\title{
Stability and Metastability of Clusters in a Reactive Atmosphere: Theoretical Evidence for Unexpected Stoichiometries of $\operatorname{Mg}_{M} \mathrm{O}_{x}$
}

\author{
Saswata Bhattacharya, Sergey V. Levchenko, Luca M. Ghiringhelli, and Matthias Scheffler \\ Fritz-Haber-Institut der Max-Planck-Gesellschaft, Faradayweg 4-6, D-14195 Berlin, Germany
}

(Received 5 April 2013; published 25 September 2013)

\begin{abstract}
By applying a genetic algorithm and $a b$ initio atomistic thermodynamics, we identify the stable and metastable compositions and structures of $\mathrm{Mg}_{M} \mathrm{O}_{x}$ clusters at realistic temperatures and oxygen pressures. We find that small clusters $(M \lesssim 5)$ are in thermodynamic equilibrium when $x>M$. The nonstoichiometric clusters exhibit peculiar magnetic behavior, suggesting the possibility of tuning magnetic properties by changing environmental pressure and temperature conditions. Furthermore, we show that density-functional theory with a hybrid exchange-correlation functional is needed for predicting accurate phase diagrams of metal-oxide clusters. Neither a (sophisticated) force field nor density-functional theory with (semi)local exchange-correlation functionals is sufficient for even a qualitative prediction.
\end{abstract}

DOI: 10.1103/PhysRevLett.111.135501

PACS numbers: $61.48 .-\mathrm{c}, 82.33 . \mathrm{Fg}, 82.65 .+\mathrm{r}$

In the search for novel functional materials, atomic (sub) nanometer clusters are widely studied as model systems exhibiting unique size-dependent properties often even qualitatively different from bulk materials. For example, small clusters may exhibit completely new local structures, stoichiometries, and electronic and magnetic properties unknown in the bulk materials [1]. Heterogeneous catalysis is just one important example where all mentioned issues are of fundamental importance [2-8].

The composition and structure of clusters are determined by thermodynamics and kinetics at the relevant temperature $(T)$ and the nature of the environment. In thermodynamic equilibrium, only structures and compositions that minimize the free energy of the combined gas + cluster system will be stable. Although a system is often not in thermodynamic equilibrium, thermodynamic phase diagrams serve as guidelines and important limits for predicting properties and functions of real materials. In this Letter, we address the issue of stability and metastability using a model system that is relevant for many practical applications: free metal $(\mathrm{Mg})$ clusters in an oxygen atmosphere.

Most of the previous research on clusters focused on properties of stoichiometric $\left(\mathrm{Mg}_{M} \mathrm{O}_{x}, x=M\right)$ clusters [7,9-17], and only a few attempts have been made to study properties of the nonstoichiometric $(x \neq M)$ clusters $[8,9,15]$. However, the decisive issue of stability and metastability of clusters with different compositions at realistic conditions (exchange of atoms with an environment) has not been addressed so far. We consider a wide range of $\mathrm{Mg}_{M} \mathrm{O}_{x}$ cluster sizes: $1 \leq M \leq 15$ and $x$ determined by thermal equilibrium with the environment at given

Published by the American Physical Society under the terms of the Creative Commons Attribution 3.0 License. Further distribution of this work must maintain attribution to the author(s) and the published article's title, journal citation, and DOI. temperature $T$ and partial oxygen pressure $p_{\mathrm{O}_{2}}$. For each stoichiometry, the energy is minimized with respect to both geometry and spin state. Unexpectedly, our results reveal (see Fig. 1) that nonstoichiometric clusters with $x>M$ are more stable at realistic $\left(T, p_{\mathrm{O}_{2}}\right)$ when $M<5$, while for bigger clusters, there is a competition between stoichiometric and more-than-stoichiometric composition $(x>M)$.

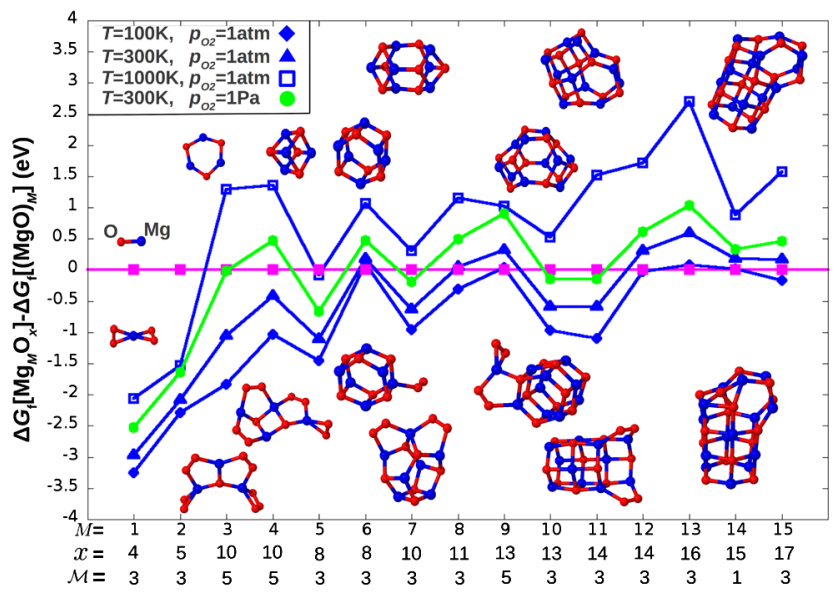

FIG. 1 (color online). Free energy of formation of thermodynamically most stable nonstoichiometric clusters $\left(\mathrm{Mg}_{M} \mathrm{O}_{x}\right.$ with $M \neq x)$, relative to stoichiometric $(M=x)$ clusters, at several $\left(T, p_{\mathrm{O}_{2}}\right)$ conditions. The geometries were optimized with PBE + $\mathrm{vdW}$, the (harmonic) vibrational free energy was evaluated with the same functional, and the electronic energy was calculated using PBE0 $+v d W$. The label of the horizontal axis shows the amount $M$ of $\mathrm{Mg}$ atoms, and the amount $x$ of $\mathrm{O}$ atoms and spin multiplicity $\mathcal{M}$ for the nonstoichiometric $\mathrm{Mg}_{M} \mathrm{O}_{x}$ cluster thermodynamically most stable at $p_{\mathrm{O}_{2}}=1 \mathrm{~atm}$ and $T=300 \mathrm{~K}$. (The stoichiometric clusters are all singlets, i.e., $\mathcal{M}=1$ ). The structure of clusters at selected sizes is also shown. (All structures are shown in the Supplemental Material [21].) 
For the determination of low-energy structures, we use a genetic algorithm (GA). The GA mimics the process of "natural" selection to evolve a pool of atomic structures until the structures that fit best the chosen selection criteria are found [18-20]. In this work, we search for structures that minimize the density-functional theory (DFT) total energy within each stoichiometry. Note that the GA is not a single method, but a whole class of methods, and must be optimized and validated for each system. Details of our implementation of the GA are given in the Supplemental Material [21], and its validation is discussed below.

The free energy as a function of $T$ and $p_{\mathrm{O}_{2}}$ is calculated for the minimum of the potential-energy surface (global minimum, GM) and (energetically) adjacent local-energy minima for each stoichiometry using the ab initio atomistic thermodynamics approach [22-25], recently extended to cluster systems $[26,27]$. The thermodynamic phase diagram is then constructed by selecting cluster compositions and structures with the lowest free energy as a function of $\left(T, p_{\mathrm{O}_{2}}\right)$.

The reliability of predictions on the relative stability of clusters with different structures depends on the accuracy of the underlying potential-energy surface (PES). Here, we find that comparing clusters with different stoichiometry poses an additional challenge, which is not apparent if only one stoichiometry is considered. Figure 2 shows a comparison of DFT energies of the reaction $\mathrm{MgO}_{x}+\mathrm{O}_{2} \rightarrow$ $\mathrm{MgO}_{x+2}$ calculated with different exchange-correlation functionals: generalized gradient approximation PBE [28] and hybrid PBE0 [29], both corrected for the

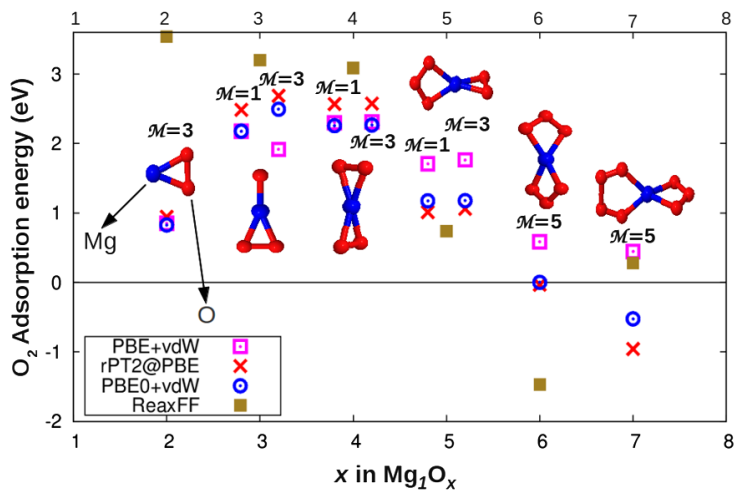

FIG. 2 (color online). Energy of $\mathrm{O}_{2}$ adsorption on $\mathrm{MgO}_{x}$ clusters (energy of the reaction $\mathrm{MgO}_{x}+\mathrm{O}_{2} \rightarrow \mathrm{MgO}_{x+2}$ calculated at the PBE+vdW GM geometry) calculated with different exchange-correlation functionals and reaxFF [38]. For the cases where singlet $(\mathcal{M}=1)$ and triplet $(\mathcal{M}=3)$ spin states are almost degenerate, the two sets of energies are shown (for DFT only, since reaxFF does not describe spin). In these cases, the spin state of $\mathrm{MgO}_{x}$ is chosen to be singlet for triplet $\mathrm{MgO}_{x+2}$, and triplet for singlet $\mathrm{MgO}_{x+2}$, to preserve the spin-conservation rule $\left(\mathrm{O}_{2}\right.$ is always kept in its ground triplet electronic state). The geometry differences of clusters with different spin states are invisible at this scale. van der Waals (vdW) interaction (PBE + vdW and PBE0 + vdW), and the highest level currently achievable within the DFT framework, i.e., the renormalized secondorder perturbation theory (rPT2) [30], here applied on PBE orbitals (rPT2@PBE). All DFT calculations are performed with the all-electron FHI-aims package, employing numerically tabulated atomic orbitals [31]. It can be seen that PBE $+v d W$ strongly overestimates the stability of clusters with larger $x$, resulting in a qualitatively incorrect prediction that $\mathrm{O}_{2}$ adsorption would be favored over desorption up to a large excess of oxygen. Such behavior is not confirmed by the PBE0 + vdW hybrid functional or rPT2@PBE (note that a similar behavior is found for PBE compared to PBE0, i.e., without vdW correction).

Interestingly, for lower $\mathrm{O}_{2}$ coverage, the difference between PBE + vdW and PBE0 + vdW/rPT2@PBE energies of $\mathrm{O}_{2}$ adsorption on $\mathrm{Mg}$ and $\mathrm{MgO}_{2}$ is small despite the error in the $\mathrm{O}_{2}$ binding energy (see Fig. 2; the calculated $\mathrm{O}_{2}$ binding energy is $6.23 \mathrm{eV}$ for $\mathrm{PBE}+\mathrm{vdW}, 5.36$ for PBE0 + vdW, 4.59 for rPT2@PBE, and the experimental value is 5.21 [32]). This can be explained by error cancelation for the clusters: If an $\mathrm{O}_{2}$ molecule adsorbs nondissociatively on $\mathrm{Mg}_{M} \mathrm{O}_{x}$, the error in the description of $\mathrm{Mg}_{M} \mathrm{O}_{x+2}$ will cancel with the error in the description of $\mathrm{O}_{2}$ when calculating the adsorption energy. Indeed, we find that adsorption of $\mathrm{O}_{2}$ on $\mathrm{Mg}$ and $\mathrm{MgO}_{2}$ does not lead to breaking of $\mathrm{O}-\mathrm{O}$ bonds. The difference between $\mathrm{PBE}+\mathrm{vdW}$ and $\mathrm{PBE} 0+\mathrm{vdW}$ energies of $\mathrm{O}_{2}$ adsorption on $\mathrm{MgO}$ for the triplet case is due to the difference in the description of the singlet state of $\mathrm{MgO}$ itself. For clusters with $x \geq 5$, correction of the $\mathrm{O}_{2}$ binding-energy error with the experimental value increases the difference between PBE + vdW and PBE0 + vdW/rPT2@PBE adsorption energies (see the Supplemental Material [21]). The tendency of PBE (and, as a consequence, of PBE $+\mathrm{vdW}$ ) to overbind $\mathrm{O}_{2}$ molecules at high coverage holds also at larger $M$ (see the Supplemental Material [21]).

It has been recently shown [33] that the HSE06 functional [34] yields a good description of the level alignment and electron transfer in $\mathrm{MgO}$. Based on comparison for selected clusters, we find that PBE0, which belongs to the same family of functionals, yields formation energies essentially identical to HSE06 (within $0.05 \mathrm{eV}$ for $\mathrm{Mg}_{M} \mathrm{O}_{x}$ neutral clusters). Taking into account this comparison and the fact that PBE0 $+\mathrm{vdW}$ results are in general much closer to rPT2@PBE than PBE + vdW, we conclude that reducing the self-interaction error by including HartreeFock exchange in DFT is crucial for correct description of the $\mathrm{Mg}_{M} \mathrm{O}_{x}$ cluster energetics, both within each stoichiometry and in particular when comparing different stoichiometries.

Therefore, the PBE0 + vdW functional is used to calculate the energies and evaluate the fitness during GA runs. In order to improve the efficiency of the GA scan, our implementation proceeds in terms of a cascade in which successive steps employ higher levels of theory, with each next level using information obtained at the lower level. 
Initially, a local optimization of a given structure is performed with lower-level (computationally relatively cheap) numerical settings. At this level, PBE + vdW and "light" numerical settings with basis set "tier 1" were used [31], and forces were converged to better than $10^{-3} \mathrm{eV} / \AA ̊$. Next, structures with energies within $2.5 \mathrm{eV}$ from the current GM candidate are further relaxed using higher-level settings [35]. We use the PBE + vdW functional with "tight- tier 2" numerical and basis settings for energy minimization at the higher level, and forces were converged to better than $10^{-5} \mathrm{eV} / \AA$. Next, the energies of these further optimized structures are reevaluated using PBE0 + vdW and "tight-tier 2" settings.

A challenging problem of any random-walk-type multidimensional global minimization scheme (including basin hopping and GA) is to guarantee that the lowest-energy structure found by the algorithm is indeed the GM. We address this validation problem by applying replicaexchange molecular dynamics [26,36,37], a (computationally very expensive) reference method that performs the canonical sampling of a PES simultaneously at different temperatures and is exhaustive if the system is ergodic. The validation of GA is performed using a reactive force field (reaxFF $[38,39]$ ) to evaluate energy and forces. While the force field is by far not accurate enough to predict correct energy differences, as can be seen in Fig. 2 and in the Supplemental Material [21], using it for the validation is a more stringent test for the GA, since the force-field PES is found to have a much more complicated landscape, with many more local minima, than the $a b$ initio PES. With our implementation of GA, we find, for the systems here considered, the same GM as found by $1.5-\mu$ s-long (cumulative time) replica-exchange molecular dynamics [40] runs. An independent evidence of the robustness of our GA scheme is that, for stoichiometric clusters, we could always find the GM reported in literature $[3,17]$.

At given $T, p_{\mathrm{O}_{2}}$, and $M$, the stable stoichiometry of a $\mathrm{Mg}_{M} \mathrm{O}_{x}$ cluster is determined via ab initio atomistic thermodynamics, i.e., by minimizing the Gibbs free energy of formation [27]: $\Delta G_{f}\left(T, p_{\mathrm{O}_{2}}\right)=F_{\mathrm{Mg}_{M} \mathrm{O}_{x}}(T)-F_{\mathrm{Mg}_{M}}(T)-$ $x \mu_{\mathrm{O}}\left(T, p_{\mathrm{O}_{2}}\right)$. Here, $F_{\mathrm{Mg}_{M} \mathrm{O}_{x}}(T)$ and $F_{\mathrm{Mg}_{M}}(T)$ are the Helmholtz free energies of the $\mathrm{Mg}_{M} \mathrm{O}_{x}$ and the pristine $\mathrm{Mg}_{M}$ cluster (at their ground state with respect to geometry and spin), respectively, and $\mu_{\mathrm{O}}\left(T, p_{\mathrm{O}_{2}}\right)$ is the chemical potential of oxygen. $F_{\mathrm{Mg}_{M} \mathrm{O}_{x}}(T)$ and $F_{\mathrm{Mg}_{M}}(T)$ are calculated using DFT information and are expressed as the sum of DFT total energy, DFT vibrational free energy in the harmonic approximation, as well as translational, rotational, and symmetry- and spin-degeneracy free-energy contributions. The dependence of $\mu_{\mathrm{O}}$ on $T$ and $p_{\mathrm{O}_{2}}$ is calculated using the ideal (diatomic) gas approximation with the same DFT functional as for the clusters [27]. Note that $\mu_{\mathrm{O}}\left(T, p_{\mathrm{O}_{2}}\right)$ is in turn a sum [27] of the same free-energy contributions as for the free clusters, where the $p_{\mathrm{O}_{2}}$ dependence is captured by the translational freeenergy term, i.e., $k_{B} T \ln p_{\mathrm{O}_{2}}+f(T)$ (where the second

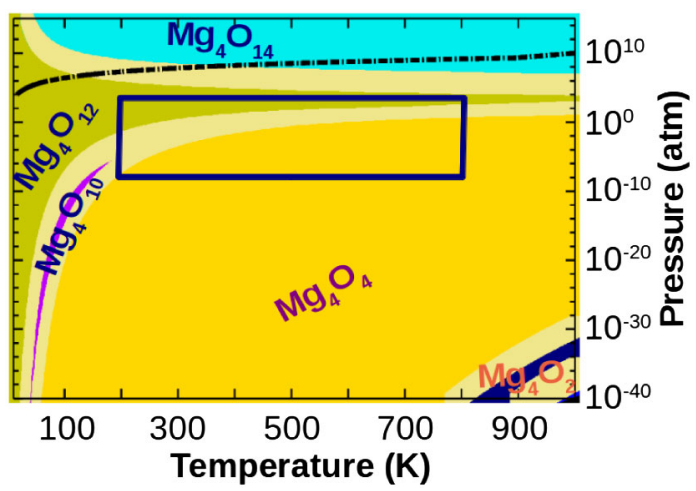

FIG. 3 (color online). Phase diagram for $\mathrm{Mg}_{4} \mathrm{O}_{x}$ clusters in an oxygen atmosphere. The geometries are optimized with $\mathrm{PBE}+$ $v d W$, (harmonic) free energies are evaluated with the same functional, and the total energies are calculated with PBE0 + $\mathrm{vdW}$. The sand-colored unlabeled areas are regions where different compositions (at least the adjacent ones) coexist (see the text). The thick black line is the O-rich limit [24,25]: Above this line, $\mathrm{O}_{2}$ droplets condense on the clusters. The rectangle encompasses a region accessible to experiments.

term does not depend on $p_{\mathrm{O}_{2}}$ ). The phase diagram for a particular $M$ is constructed by identifying the lowest-freeenergy structures at each $\left(T, p_{\mathrm{O}_{2}}\right)$. As a representative example, we show in Fig. 3 the phase diagram for $M=4$. Phase diagrams based on reaxFF, PBE + vdW, PBE0 (without vdW), and rPT2@PBE can be found in the Supplemental Material [21]. We find that at all DFT levels, the phase diagrams are qualitatively and quantitatively very similar for $T>200 \mathrm{~K}$ and $p_{\mathrm{O}_{2}}<10^{-5} \mathrm{~atm}$. For higher pressures and/or lower temperatures, however, $\mathrm{PBE}+\mathrm{vdW}$ predicts larger $x$ in $\mathrm{Mg}_{M} \mathrm{O}_{x}$ as thermodynamically more stable, compared to PBE $+\mathrm{vdW}$ and rPT2@PBE. This is consistent with the results shown in Fig. 2; i.e., PBE tends to favor adsorption of a larger number of $\mathrm{O}_{2}$ molecules. PBE0 and PBE0 + vdW yield almost identical phase diagrams. Thus, at the considered cluster sizes, the vdW interactions, within the scheme of Ref. [41], do not affect the differences between free energies of formation of competing $\mathrm{Mg}_{M} \mathrm{O}_{x}$ clusters. ReaxFFbased diagrams were evaluated for the sake of comparison: they are very different from DFT-based ones, and no conclusions, even qualitative ones, can be drawn from the analysis of the reactive force-field results.

For creating the phase diagrams, we have approximated the configurational free energy with the harmonic vibrational free energy. If the clusters exhibit fluxional behavior or are melted, as it is the case for some metal clusters, this approximation can become invalid at high or even moderate temperatures [26,42]. For all the $\operatorname{Mg}_{M} \mathrm{O}_{x}$ clusters described here, we have tested the validity of the harmonic assumption by running ab initio molecular dynamics (MD) simulations for $20 \mathrm{ps}$ at $T=800 \mathrm{~K}$ [43]. We found that, while the structures exhibit large vibrations, their bond connectivity is never destroyed, and that the initial $0 \mathrm{~K}$ 
structure was reversibly recovered by cooling down the hot sample. At most, some of the $\mathrm{O}_{2}$ moieties (see below) either freely rotate or roam around the clusters at larger $x$. In order to quantitatively account for these larger displacements in terms of configurational entropy, we have evaluated the excess free energy of selected clusters by integrating the thermodynamic relation $\partial(\beta F(\beta)) / \partial \beta=$ $\langle U\rangle_{\beta}$, where $\langle U\rangle_{\beta}$ is the canonical average of the total energy at temperature $T=1 / k_{B} \beta$. The differential equation is numerically integrated by evaluating $\langle U\rangle_{\beta}$ via 4-pslong (after equilibration) MD trajectories at five increasing temperatures, from $T=10 \mathrm{~K}$ (where the reference free energy is safely approximated by the harmonic expression) to $T=800 \mathrm{~K}$. The integration path was checked to be reversible. In this way, all anharmonic contributions are included through the canonical sampling of the PES. For the $\mathrm{Mg}_{4} \mathrm{O}_{x}$, the anharmonic correction enlarges the stability region at higher temperatures of the highest stoichiometry $\mathrm{Mg}_{4} \mathrm{O}_{12}$ by at most $50 \mathrm{~K}$ at $p_{\mathrm{O}_{2}}=1 \mathrm{~atm}$. Note that the (harmonic or beyond) configurational free energy is only one part of the total free energy and that, at higher $(T$, $p_{\mathrm{O}_{2}}$ ) and stoichiometries $x$, the free energy is dominated by the term $x k_{B} T \ln p$. For this reason, the plots are extended up to $1000 \mathrm{~K}$, where weakly bound $\mathrm{O}_{2}$ moieties may evaporate; nonetheless, at thermodynamic equilibrium, the pressure of $\mathrm{O}_{2}$ molecules ensures that another $\mathrm{O}_{2}$ molecule will on average replace the evaporated one. Furthermore, we find (see the Supplemental Material [21]) that neglecting translational, rotational, (harmonic or beyond) vibrational, and symmetry- and spin-degeneracy-related free-energy contributions results only in slight changes in the phase diagrams, comparable to the differences between PBE0+vdW and rPT2@PBE diagrams, for all considered $M$.

We have constructed phase diagrams for $1 \leq M \leq 15$. At thermodynamic equilibrium in a wide range around normal conditions, small $\mathrm{Mg}_{M} \mathrm{O}_{x}$ clusters $(M \leq 5)$ are found to be preferentially nonstoichiometric $(x>M)$. For sizes $M>5$, we observe a competition between stoichiometric and nonstoichiometric stable structures. In Fig. 1, we also report the relative stability of stoichiometric and nonstoichiometric structures at low $(T=100 \mathrm{~K})$ and high $(T=1000 \mathrm{~K})$ temperatures. The relative stability of stoichiometric structures increases with temperature.

A further interesting property we find is that $\mathrm{Mg}_{M} \mathrm{O}_{x}$ nonstoichiometric clusters have in general several neardegenerate (within $\sim 0.05 \mathrm{eV}$ ) electronic states with different spin. The highest multiplicity we predict is $\mathcal{M}=7$ for the case $\mathrm{Mg}_{4} \mathrm{O}_{12}$, and values of $\mathcal{M}=3$ and 5 are largely represented. The analysis of the spin density shows that the unpaired electron density is localized on $\mathrm{O}_{2}$ and $\mathrm{O}_{3}$ moieties (see examples in the Supplemental Material [21]). The orbitals occupied by the different unpaired electrons in the same cluster have vanishing overlaps due to large separation. The different spin states for each isomer can be formed and coexist in oxygen atmosphere without violating spin-conservation rules, since successive adsorption and desorption of the (triplet) $\mathrm{O}_{2}$ molecules in the gas phase allows the clusters to reach all the stable spin multiplicities observed for each cluster. The thermodynamically favored access of oxygen in small clusters is in sharp contrast to bulk pristine $\mathrm{MgO}$, where stoichiometric composition is strongly favored. In all cases where $\mathrm{O}_{2}$ or $\mathrm{O}_{3}$ moieties are coordinated to maximum two $\mathrm{Mg}$ atoms, these moieties host an unpaired spin. When the number of $\mathrm{Mg}$ is increased such that every $\mathrm{O}$ atom can be coordinated to three or more $\mathrm{Mg}$, and at the same time the clusters have large HOMO-LUMO gaps (i.e., all valence $\mathrm{Mg}$ electrons are transferred to $\mathrm{O}_{x}$ ), then stoichiometric clusters become thermodynamically stabilized.

All the quasidegenerate spin states are populated at finite temperature; thus, all the nonstoichiometric clusters with energetically quasidegenerate states are paramagnetic. In contrast to nonstoichiometric clusters, we find that stoichiometric structures are always singlet, separated from the higher-multiplicity states by at least $1 \mathrm{eV}$. Thus, the stoichiometric clusters are diamagnetic. By looking at Fig. 3, we note that in the range of realistically achievable pressures encompassed by the rectangle, nonstoichiometric (paramagnetic) structures are thermodynamically more stable at lower temperatures, while at higher temperatures, the stoichiometric (diamagnetic) structures become more stable. The temperature at which this transition occurs is a rapidly varying function of $p_{\mathrm{O}_{2}}$. We have thus identified a class of systems that undergo an unusual paramagneticdiamagnetic transition induced by $T$ and $p$ of the reactive atmosphere, where the change in magnetic behavior reflects the change in composition. The transition between paramagnetic and diamagnetic behavior is smooth when environmental conditions are changed smoothly because different stoichiometries always coexist within a few $k_{B} T$. In fact, the sand-colored areas in Fig. 3 are the regions where the free energies of the competing compositions or structures are within an energy range of $2 k_{B} T$.

In conclusion, we have presented a theoretical framework for predicting the structure and stoichiometry of stable and metastable clusters in thermodynamic equilibrium with a gas atmosphere. An efficient and unbiased scan of the potential energy of the clusters at various compositions is combined with ab initio atomistic thermodynamics, a tool for evaluating the relative free energy of structures by knowing their electronic energy, vibrational frequencies, and structural parameters for the evaluation of translational and rotational entropy. The methodology has been applied to $\mathrm{Mg}$ clusters in an oxygen atmosphere. We have shown that small alkaline earth metal clusters form thermodynamically stable nonstoichiometric "nano-oxides," which have been overlooked so far. They are also predicted to have peculiar, $(T, p)$-dependent, magnetic properties.

We acknowledge the cluster of excellence "Unifying Concepts in Catalysis" (UniCat, sponsored by the DFG and administered by the TU Berlin) for financial support. 
[1] E. Roduner, Chem. Soc. Rev. 35, 583 (2006).

[2] K. G. Caulton, M. G. Thomas, B. A. Sosinsky, and E. L. Muetterties, Proc. Natl. Acad. Sci. U.S.A. 73, 4274 (1976).

[3] K. Kwapien, M. Sierka, J. Dobler, J. Sauer, M. Haertelt, A. Fielicke, and G. Meijer, Angew. Chem., Int. Ed. Engl. 50, 1716 (2011).

[4] N. Marom, M. Kim, and J. R. Chelikowsky, Phys. Rev. Lett. 108, 106801 (2012).

[5] Y. Gao, N. Shao, Y. Pei, Z. Chen, and X.C. Zeng, ACS Nano 5, 7818 (2011).

[6] Yimin Li et al., J. Am. Chem. Soc. 133, 13527 (2011).

[7] W. A. Saunders, Phys. Rev. B 37, 6583 (1988).

[8] T. Uchino and T. Yoko, Phys. Rev. B 85, 012407 (2012).

[9] P. J. Ziemann and A. W. Castleman, Jr., Phys. Rev. B 44, 6488 (1991).

[10] S. Moukouri and C. Noguera, Z. Phys. D 24, 71 (1992).

[11] S. Moukouri and C. Noguera, Z. Phys. D 27, 79 (1993).

[12] J. M. Recio and R. Pandey, Phys. Rev. A 47, 2075 (1993).

[13] M.-J. Malliavin and C. Coudray, J. Chem. Phys. 106, 2323 (1997).

[14] E. de la Puente, A. Aguado, A. Ayuela, and J. M. Lopez, Phys. Rev. B 56, 7607 (1997).

[15] C. Roberts and R. L. Johnston, Phys. Chem. Chem. Phys. 3, 5024 (2001).

[16] A. Jain, V. Kumar, M. Sluiter, and Y. Kawazoe, Comput. Mater. Sci. 36, 171 (2006).

[17] M. Haertelt, A. Fielicke, G. Meijer, K. Kwapien, M. Sierkaz, and J. Sauer, Phys. Chem. Chem. Phys. 14, 2849 (2012).

[18] R. L. Johnston, Dalton Trans. 22, 4193 (2003).

[19] M. Sierka, Prog. Surf. Sci. 85, 398 (2010).

[20] L. B. Vilhelmsen and B. Hammer, Phys. Rev. Lett. 108, 126101 (2012).

[21] See Supplemental Material at http://link.aps.org/ supplemental/10.1103/PhysRevLett.111.135501 for details on thermodynamic stability of stoichiometric vs nonstorichiometric $\mathrm{Mg}_{M} \mathrm{O}_{\mathrm{X}}$ clusters; implementation of GA; performance of reaxFF; $\mathrm{O}_{2}$-adsorption energy on $\mathrm{MgO}_{\mathrm{X}}$ with functionals corrected by the experimental value of $\mathrm{O}_{2}$ binding energy; $\mathrm{O}_{2}$-adsorption energy on $\mathrm{Mg}_{2} \mathrm{O}_{\mathrm{X}}$ and $\mathrm{Mg}_{3} \mathrm{O}_{\mathrm{X}}$ clusters, $\mathrm{Mg}_{2} \mathrm{O}_{\mathrm{X}}$ phase diagrams with various functionals; effect of translational, rotational, vibrational contributions to the free energy on $\mathrm{Mg}_{2} \mathrm{O}_{\mathrm{X}}$ phase diagram; effect of anharmonic contributions to configurational free energy; and examples of spin densities on nonstochiometric $\mathrm{Mg}_{M} \mathrm{O}_{\mathrm{X}}$.

[22] C.M. Weinert and M. Scheffler, in Defects in Semiconductors, edited by H.J. von Bardeleben (Trans Tech Publications Ltd, Switzerland, 1986), pp. 25-30.

[23] M. Scheffler and J. Dabrowski, Philos. Mag. A 58, 107 (1988).

[24] K. Reuter and M. Scheffler, Phys. Rev. B 65, 035406 (2001); Phys. Rev. B 75, 049901(E) (2007).

[25] K. Reuter, C. Stampfl, and M. Scheffler, in Handbook of Materials Modeling, edited by S. Yip (Springer, New York, 2005), p. 149.

[26] E. C. Beret, L. M. Ghiringhelli, and M. Scheffler, Faraday Discuss. 152, 153 (2011).

[27] E. C. Beret, M. van Wijk, and L. M. Ghiringhelli, Int. J. Quantum Chem., doi:10.1002/qua.24503 (2013).

[28] J. P. Perdew, K. Burke, and M. Ernzerhof, Phys. Rev. Lett. 77, 3865 (1996); Phys. Rev. Lett. 78, 1396 (1997).

[29] J. P. Perdew, K. Burke, and M. Ernzerhof, Phys. Rev. Lett. 77, 3865 (1996).
[30] X. Ren, A. Tkatchenko, P. Rinke, and M. Scheffler, Phys. Rev. Lett. 106, 153003 (2011); X. Ren, P. Rinke, C. Joas, and M. Scheffler, J. Mater. Sci. 47, 7447 (2012); X. Ren, P. Rinke, G. E. Scuseria, and M. Scheffler, Phys. Rev. B 88, 035120 (2013).

[31] V. Blum, R. Gehrke, F. Hanke, P. Havu, V. Havu, X. Ren, K. Reuter, and M. Scheffler, Comput. Phys. Commun. 180, 2175 (2009); PBE and PBE0 energies were calculated using "tight-tier 2" numerical and basis settings. rPT2@PBE energies were calculated using "really tight" grid settings and a "tier 4" basis set, and counterpoise corrected for the basis set superposition error.

[32] M.W. Chase, Jr., J. Phys. Chem. Ref. Data Monogr. 9, 1 (1998); http://webbook.nist.gov/cgi/cbook.cgi? ID=C7782447; zero-point energy correction taken from K. P. Huber and G. Herzberg, in Molecular Spectra and Molecular Structure: Constants of Diatomic Molecules (Van Nostrand Reinhold, New York, 1979), Vol. 4.

[33] N. A. Richter, S. Sicolo, S. V. Levchenko, J. Sauer, and M. Scheffler, Phys. Rev. Lett. 111, 045502 (2013).

[34] A. V. Krukau, O. A. Vydrov, A.F. Izmaylov, and G. E. Scuseria, J. Chem. Phys. 125, 224106 (2006).

[35] A two-level GA was proposed in Ref. [20]; however, our cascade approach is developed into a wider scheme (see the Supplemental Material [21]).

[36] E. Marinari and G. Parisi, Europhys. Lett. 19, 451 (1992).

[37] D. J. Sindhikara, D. J. Emerson, and A.E. Roitberg, J. Chem. Theory Comput. 6, 2804 (2010).

[38] A. C. T. van Duin, S. Dasgupta, F. Lorant, and W. A. Goddard, J. Phys. Chem. A 105, 9396 (2001). The reaxFF is probably the best reactive FF, for which a parametrization exists for a broad class of materials. The reaxFF parameters we have used for $\mathrm{Mg}$ and $\mathrm{O}$ [39] were fit [39] to a quantum-mechanics training set including $\mathrm{MgO}$-bulk and $\mathrm{O}_{2}$-molecule properties. We checked that this parametrization yields for the bulk $\mathrm{MgO}$ lattice constant within $1 \%$ and bulk modulus within $10 \%$ from the experimental values; similarly, for the $\mathrm{MgO}$ and $\mathrm{O}_{2}$ dimer, it gives bond length and vibrational frequency within $1 \%$ and $10 \%$ from the respective reference values. On this set of properties, reaxFF performs remarkably well, comparably to $\mathrm{PBE}+\mathrm{vdW}$ [28], $\mathrm{PBE0}+\mathrm{vdW}$ [29], and HSE06 + vdW [34], however its range of validity is found to be rather narrow, in fact it fails very clearly for small clusters. In more detail, reaxFF yields a qualitatively good prescanning for stoichiometric clusters, while for nonstoichiometric ones, it is far from desirable accuracy. This is not unexpected, as not even PBE is able to account for the charge redistributions associated with nonstoichiometric clusters.

[39] R. Zhu, F. Janetzko, Y. Zhang, A. C. T. van Duin, W. A. Goddard, and D. R. Salahub, Theor. Chim. Acta 120, 479 (2008).

[40] S. Bhattacharya, S. V. Levchenko, L. M. Ghiringhelli, and M. Scheffler (to be published).

[41] A. Tkatchenko and M. Scheffler, Phys. Rev. Lett. 102, 073005 (2009).

[42] P. Koskinen, H. Häkkinen, B. Huber, B. von Issendorff, and M. Moseler, Phys. Rev. Lett. 98, 015701 (2007).

[43] The Born-Oppenheimer MD simulations were performed with the PBE functional, tight-tier 2 settings, a 1 fs time step, and a stochastic velocity-rescaling thermostat. 